WILENMANN, Javier: "El derecho frente a la resistencia a la criminalización bajo el ejemplo de los delitos de corrupción".

Polít. Crim. Vol. 15, No 29 (Julio 2020), Art. 11, pp. 310-330

[http://politcrim.com/wp-content/uploads/2020/07/Vol15N29A11.pdf]

\title{
El derecho frente a la resistencia a la criminalización bajo el ejemplo de los delitos de corrupción
}

\section{The Law in the Face of Resistance to Criminalization. The Example of Corruption Offenses}

\author{
Javier Wilenmann von Bernath* \\ Dr. Iur. Albert-Ludwig Universität Freiburg \\ Profesor Facultad de Derecho Universidad Adolfo Ibáñez \\ javier.wilenmann@uai.cl
}

\section{Resumen}

El artículo tematiza un problema central que aqueja a la reacción jurídico-penal frente a actos de corrupción de alto nivel: la resistencia a la criminalización y a la consideración como corrupta de acciones desarrolladas por individuos de alto status en la economía ordinaria. Su objetivo es demostrar el rendimiento que tiene leer algunas características de los delitos de corrupción a partir de este fenómeno. Ello permite, entre otras cosas, reconocer y someter a análisis tres técnicas de reacción frente al fenómeno: la tabuización, la persecución oblicua y la regulación por indeterminación.

Palabras claves: Corrupción, cohecho, soborno, criminología de cuello y corbata, persecución oblicua, indeterminación

\begin{abstract}
The paper analyzes a central issue in the legal enforcement of bribery and other anticorruption offenses: white-collar resistance to criminalization and corruption-labeling. It aims to show the uses of understanding corruption criminal law under the prism of resistance, including the identification and analysis of three modes of reaction to resistance: taboogeneration, pretextual prosecution and regulation through legal indeterminacy.
\end{abstract}

Keywords: Corruption, bribery, White-collar criminology, pretextual prosecution, indeterminacy

\section{Introducción}

Varios de los casos de corrupción que han sido conocidos durante los últimos años en Chile, y que han impulsado reformas penales sustanciales en el último tiempo, comparten características que no son difíciles de identificar. Sea que se trate del uso de fondos

\footnotetext{
* Una versión preliminar de este artículo fue presentada en un seminario organizado en la Universidad de Valparaíso por el profesor José Luis Guzmán Dalbora. Agradezco a todos los participantes por sus comentarios y sugerencias. El artículo forma parte del proyecto Fondecyt $\mathrm{N}^{\circ} 1170056$ del que soy el investigador responsable.
} 
reservados para financiar gastos personales, de otorgamientos de boletas de prestación de servicios para cubrir donaciones políticas, o de otras acciones similares, en ellas personas de alto estatus dentro de una organización o de un sector social desarrollaban prácticas más o menos normalizadas y, en algunos casos, ampliamente conocidas también por externos. La explicación: "esto era una práctica conocida", "todos hacían esto", es frecuente en la reacción a estos casos. Y, en algo tal vez más sorprendente, esas reacciones tendían a tener lugar a una vez que la práctica normalizada devino en su contrario, el escándalo.

Descrito en términos más generales, la corrupción de alto nivel tiende a insertarse en prácticas que cuentan con relativa aceptación en los sectores en los que se desarrollan, incluyendo a los agentes de control del sector en cuestión. Al ser expuestas, sin embargo, a ciertas formas de control social externo (ej.: prensa), la reacción frente a la práctica se transforma -ante todo en el caso de corrupción con involucramiento político ${ }^{1}$ - con alta probabilidad en escándalo. La corrupción de alto nivel presenta, en ese sentido, una dinámica que desde el punto de vista de los implicados puede parecer esquizofrénica: a la normalización le sigue violentamente el escándalo.

En esas condiciones, no es inusual que la reacción de los involucrados sea de incomprensión. ${ }^{2}$ Tal vez las prácticas en cuestión se encontraban "al límite" o eran "grises" (por ello se realizaban de modo más o menos clandestino), tal vez se revelaran después a sus participantes como "éticamente reprochables", pero en cualquier caso se habrían insertado en la "legalidad". O, en el peor de los casos, se trataría de faltas administrativas, de problemas tributarios a ser resueltos por medio del cobro de ciertos impuestos que se revelaron como devengados y no pagados. Pero en ningún caso podría tratarse de "verdadera" corrupción ni mucho menos de delitos.

Este fenómeno, naturalmente poco advertido en estudios jurídicos sobre la corrupción y su persecución penal, es el objeto de este artículo. En particular, me interesa no solo dar cuenta de su existencia y sucintamente de posibles causas, sino que ante todo estudiar algunas de las reacciones que pueden ser reconocidas a su respecto. La tesis es sencilla: en la corrupción, al igual que en otros ámbitos de criminalidad económica, se generan efectos especialmente intensos de resistencia a la criminalización. Las fuentes de esa resistencia se manifiestan de distinto modo y empujan a distintas formas de reacción. En buena medida, entender los problemas más relevantes que aquejan a los delitos de corrupción (y a la criminalidad económica) es entender cómo el Derecho Penal reacciona frente a esa forma especial de resistencia. A la luz de los casos chilenos, tres modos de reacción se revelan como especialmente interesantes y dignos de análisis: la generación de tabús en ciertas formas de interacción, la persecución oblicua (pretextual prosecution), y la regulación por vía de indeterminación.

Para la realización de este análisis, la sección 1 se inicia dando cuenta del hallazgo de las ciencias sociales que pretendo utilizar para estos efectos, a saber, la resistencia a la criminalización (sofisticación, tendencia a la elusión) que se manifiesta en ámbitos económicos ordinarios y crecientemente a medida que avanza el estatus. A partir de la

\footnotetext{
${ }^{1}$ Sobre las razones de esta relación, véase HARRIS (2003), p. 2.

${ }^{2}$ DE GRAAF (2007), pp. 39-86.
} 


\section{Polít. Crim. Vol. 15, № 29 (Julio 2020), Art. 11, pp. 290-310 [http://politcrim.com/wp-content/uploads/2020/07/Vol15N29A11.pdf]}

descripción de este fenómeno, la sección 2 cumple una función de transición: simplemente dar cuenta del problema que supone la sofisticación para el funcionamiento del derecho como sistema de control. Las secciones 3 y 4 finalmente analizar tres formas de reacción fácilmente reconocibles frente al problema de la resistencia, centrándose en las prácticas regulatorias que denominaré tabuización (3), persecución oblicua y regulación por vía de indeterminación (4).

\section{La indeterminación del mundo ordinario como fuente de problemas jurídicos}

\subsection{El diagnóstico general de la resistencia y de la sofisticación}

Parte importante de los problemas de aplicación del Derecho Penal económico (y en general: de la regulación sancionatoria) se originan en un hallazgo criminológico relativamente trivial, a saber, la resistencia formal a la criminalización en ámbitos económicos ordinarios. ${ }^{3}$ Mientras el modo natural de resistencia en otras formas de criminalidad, incluyendo criminalidad económica de bajo nivel, consiste en esconder información sobre la perpetración del hecho, en la criminalidad económica de alto nivel la resistencia opera antes por contestación de la pretensión formal de criminalización a nivel político, social y judicial ${ }^{4}$. El hallazgo en cuestión tiene su origen en la verificación de las enormes diferencias en la prevalencia de la criminalización de las acciones de individuos de estratos bajos y altos. Ese hallazgo, por cierto, puede tener un origen causal tanto "desde abajo" 5 - la inserción en estratos bajos empuja a la desviación y a la delincuencia - como "desde arriba" - la inserción en estratos altos protege frente a la criminalización. Así, bien puede ser que las estructuras sociales generen tendencia a la delincuencia en estratos bajos por frustración relativa (relative deprivation) de expectativas materiales y simbólicas, o por razones de ecología urbana, por deficiencias de socialización, etc. Pero la pregunta también puede ser planteada al revés ${ }^{6}$ : ¿Por qué las acciones típicamente desarrolladas por individuos de estratos más altos son resistentes a la criminalización?

El fenómeno fue advertido por Edwin Sutherland ${ }^{7}$ en sus ya clásicos trabajos sobre la "criminalidad de cuello blanco": mientras la reacción a las clases de acciones que se concentran en las clases bajas, ligadas a la afectación de la propiedad privada, mantienen siempre una atribución del sentido estigmatizante asociada a la noción de crimen, la organización institucional misma de las reacciones a las acciones socialmente dañosas de personas de estratos altos, se dirigen en general a evitar esa atribución de sentido. Parte

\footnotetext{
${ }^{3}$ Por ámbitos económicos ordinarios me refiero a aquellas actividades económicas que son desarrolladas lícitamente dentro de la economía. Ello incluye a la burocracia estatal, al mundo de las empresas de distinto tamaño, al comercio lícito, etc.

${ }^{4}$ Este es el diagnóstico de TIEDEMANN (2014), pp. 735-746, aunque contraponiendo la elusión como modo criminógeno central de grandes empresas con infracciones sencillas encubiertas en criminalidad de más bajo nivel.

${ }^{5}$ SUTHERLAND (1949), cap. 1.

${ }^{6}$ BECKER (1963), pp. 13 y ss.

${ }^{7}$ SUTHERLAND (1940), pp.1-12, SUTHERLAND (1949), passim; SUTHERLAND (1945), pp. 132-39. Por cierto, aunque se trate de la fuente más conocida, la identificación del fenómeno no es un hallazgo original de Sutherland. Como denuncia ella se encontraba presente en la tradición marxista y socialista europea, y los trabajos de Thurman Arnold ya habían claramente articulado el punto. Véase ARNOLD (2010), p. 9.
} 
relevante del estudio de la "criminalidad de cuello blanco" se concentra en ese hallazgo, sus causas y consecuencias. ${ }^{8}$

El fenómeno específico que nos interesa estudiar jurídicamente - la resistencia a la criminalización en los delitos de corrupción de alto nivel - no es más que una expresión de este fenómeno general: en actividades económicas definidas como socialmente normales, es común que desviaciones más o menos obvias de los cánones normales se normalicen y sean toleradas por agentes de control formales e informales. ${ }^{9}$ Esa tendencia a la normalización, vista desde el punto de vista de los estratos beneficiados por este fenómeno, puede ser vista como expresión de su particular "sofisticación", esto es, de la capacidad de generar resistencia en los agentes de control a la consideración de sus acciones como ilícitas. ${ }^{10}$

Aunque el estudio de la sofisticación (o de la resistencia y de la elusión) tiene interés en todo el ámbito de los delitos insertos en la economía ordinaria, aquí me interesa resaltar más de cerca su relevancia en un solo ámbito de éstos, a saber, en la corrupción: tanto la dinámica general de la corrupción como la idea misma de corrupción sistémica se correlacionan directamente con el fenómeno de la sofisticación. En lo que sigue, me interesa explicar brevemente esa relación.

\subsection{La dinámica de la sofisticación en la corrupción}

La corrupción vive de la ambigüedad de la cultura. Esa ambigüedad puede ser utilizada tanto para disminuir el riesgo de criminalización en contextos de relativa incertidumbre sobre la aceptación del comportamiento por el interlocutor - en el contexto de corrupción de bajo nivel: ¿Quién pide u ofrece el soborno? ¿Cómo se comunica el ofrecimiento o la solicitud? ${ }^{11}$ -, como para evitar la concesión del sentido de acción desviada a un comportamiento determinado.

A causa del aumento progresivo de resistencia a la categorización como desviado o ilícito a medida que aumenta el status social de un grupo, el modo paradigmático de integración de la corrupción en la dinámica de la sofisticación es la corrupción sistémica de alto nivel. Precisamente por ello es que, a diferencia de la corrupción de bajo nivel, ella se encuentra extendida en todas clases de sociedades. ${ }^{12}$

Aquí es importante clarificar los usos conceptuales envueltos, ya que ellos inciden directamente sobre el objeto de estudio. Corrupción sistémica o de alto nivel es un concepto que se construye por oposición a corrupción sectorial y puramente situacional o de

\footnotetext{
${ }^{8}$ Aquí es importante no dejarse influenciar por el sentido que adquirió, con posterioridad, el concepto de Sutherland: en la actualidad se utiliza la idea de "delitos de cuello blanco" como un modo de designar al derecho penal económico. Pero el punto de Sutherland era precisamente el contrario: los delitos de cuello blanco designan a una categoría que se caracteriza por ser ciertamente reconocida como ilegal o, al menos, socialmente dañosa, pero ser resistente a la criminalización. La enorme masa de literatura que se ha generado al respecto en la literatura legal. Véase solo SHAPIRO (1990), pp. 346-365; GREEN (2004), pp. 1-34, han tendido a desconocer esto.

${ }^{9}$ COLEMAN (1987), pp. 406- 439.

${ }^{10}$ El concepto proviene de TURK (1969), p. 68.

${ }^{11}$ DE GRAAF (2008), pp. 649.; NUIJTEN y ANDERS (2007), pp. 1-26.

12 MISANGYI et al. (2008), pp. 750-70; NIELSEN (2003), pp. 125-49; WARREN (2004), pp. 328-343.
} 


\section{Polít. Crim. Vol. 15, No 29 (Julio 2020), Art. 11, pp. 290-310 [http://politcrim.com/wp-content/uploads/2020/07/Vol15N29A11.pdf]}

oportunidad (petty corruption). ${ }^{13}$ La distinción no es una de extensión: si las oportunidades para pequeños actos de corrupción son amplias, la corrupción situacional seguramente tendrá una extensión especialmente amplia. Por ejemplo, puede ser que en un lugar determinado todo policía espere que se le dé dinero para no cursar infracciones de tránsito. Si ese es el caso, es probable que se realicen frecuentemente actos de corrupción. Pero esa extensión de la corrupción no la convierte, bajo el uso conceptual aquí asumido, en sistémica o de alto nivel. Por definición, para calzar dentro del concepto, los actos en cuestión deben (i) integrarse en prácticas no oportunistas de corrupción y tener lugar a "alto nivel" (ello la distingue de la corrupción de oportunidad o petty corruption) y (ii) afectar a varias clases de interacciones (y no solo a un "sector", p.ej. a la construcción). ${ }^{14}$

La tendencia al desarrollo de la corrupción de alto nivel y a su normalización no es especialmente difícil de explicar y, de hecho, en esa simpleza se encuentra su problema. La corrupción sistémica necesita de resistencia a la consideración de las acciones en cuestión como ilícitas y, ante todo, como criminales. La sofisticación - la tendencia a la normalización y a la resistencia de las acciones económicas ordinarias de alto estrato a su consideración como desviadas - hace probable que esa resistencia tenga lugar. ${ }^{15}$ La corrupción sistémica se realiza así sobre el trasfondo de que las acciones son normalizadas por los involucrados y trabajadas por asesores que consiguen con ello reforzar la sensación de normalidad de la práctica $^{16}$.

Esa normalización se explica por todos los factores que explican la sofisticación de los estratos altos. Sin ánimo de taxatividad, ello incluye a la identidad de clase (cultural) de agentes de control y sujetos de control; el manejo de la construcción del mundo social por los estratos altos; la ambigüedad de sentido de las actividades en que se desempeñan los miembros de los estratos altos; la disposición al uso y a la explotación de esa ambigüedad en las actividades económicas ordinarias (ejemplo: disposición a la elusión de la regulación y

\footnotetext{
${ }^{13}$ Sobre la distinción entre corrupción de oportunidad (o petty corruption), corrupción de largo plazo en una relación individualizada, corrupción sectorial y corrupción sistémica, véase SCHÖN (2011), pp. 23 y ss.; DÖLLING (2007), pp. 1-40, Nm. 14 y ss.; DORSCHFELDT (2016), pp. 36 y ss. Fundamental como investigación empírica en Alemania BANNENBERG (2002), pp. 83 y ss.

${ }^{14}$ Las razones de este uso dicen relación con el tipo de investigación de que se trata aquí: como lo que interesa es el efecto de la resistencia generado por estatus y apariencia de normalidad, la extensión no es un criterio que sea de por sí relevante.

${ }^{15}$ Un punto similar pero, sin embargo, formulado de modo exactamente inverso, es levantado por USLANER, (2008), passim al vincular corrupción y desigualdad: la corrupción se generaría por la baja confianza de las clases altas en las clases bajas dadas por su propio sesgo de observación, lo que lleva a clientelismo y patronaje. Mucho más especulativa es la explicación provista por GALPERIN et al (2011), pp. 407-24: la diferenciación de estatus resultante de la estratificación al interior de una organización produciría aislamiento moral en los individuos de alto estatus y, a partir de ello, desconsideración por los demás miembros de la organización.

${ }^{16} \mathrm{El}$ fenómeno de la normalización de la corrupción se encuentra bien estudiado en distintos ámbitos de las ciencias sociales. Así, en la literatura sobre comportamiento organizacional DE GRAAF y HUBERTS (2011), passim; ANAND et al. (2004), pp. 39-53; EARLE (2010), pp, 218-37; MISANGYI et al. (2008), passim; ZYGLIDOPOULOS et al. (2009), pp. 65-73; DEN NIEUWENBOER y KAPTEIN (2008), pp. 133-46; PINTO et al. (2008), pp. 685-709. En esta literatura, es usual encontrar explicaciones de las razones por las que nudos o áreas completas de una organización generan prácticas corruptas que continúan reproduciéndose pese a los cambios en la identidad de los individuos que participan del grupo en cuestión. Observaciones similares pueden encontrarse en la psicología social (TAVITS (2010), pp. 1257-79; o en la antropología (NUIJTEN y ANDERS (2007), passim; MISANGYI et al. (2008), passim; DE GRAAF (2007), passim).
} 
de la tributación; manejo comunicacional, litigación, consultas a organismos de control y otros modos de liberación de ámbitos de acción); el uso de las instituciones para imponer intereses (litigación, "persecución política"); ${ }^{17} \mathrm{y}$ el efecto que el prestigio y el status (y probablemente la belleza) tienen en la generación de sesgos cognitivos que disponen a la normalización. ${ }^{18}$

Los factores en cuestión solo tienen por objeto ilustrar el modo en que funciona el fenómeno de la sofisticación y su vínculo con la ambigüedad de sentido que, para ellas mismas, tienen sus actividades. Aquí no me interesa producir demostraciones causales o dar cuenta representativamente de prevalencia, sino más bien mostrar la relevancia de un fenómeno, reconocido a partir de información ampliamente disponible en la literatura, para el mundo jurídico.

\section{Indeterminación y regulación}

La sofisticación y su relación con la corrupción muestran que el funcionamiento correcto del Derecho Penal, y en general de la regulación, en estos ámbitos requiere de una realidad improbable, a saber, que los agentes de los sistemas de control formal (tribunales, autoridades administrativas, en la definición de lo punible la legislación) identifiquen los ámbitos explotados de ambigüedad y eviten su uso. El diagnóstico de la sofisticación debiera servir, al menos, como advertencia: la dogmática y la práctica judicial operan aquí en un ámbito saturado por juegos de explotación de la ambigüedad; el cumplimiento de la función requiere al menos estar alerta a ello.

Jurídicamente decodificado, el problema se refiere así al control de la indeterminación. Pero la indeterminación que nos interesa no es solo relativa al contenido del derecho, sino al sentido de acciones en el mundo social. En el razonamiento jurídico, estamos acostumbrados a la producción de alternativas de argumentación para los casos en que el derecho, por su propia fijación lingüística, se ve aquejado por indeterminación de mayor o menor grado. La dogmática es, en parte, una respuesta a esa indeterminación formal. Lo que sin embargo no se puede ver sin observar la realidad judicial y sin hacer uso de conocimiento empírico es que, en el caso de la criminalidad inserta en la economía ordinaria, incluyendo a la "corrupción", la indeterminación deriva de la ambigüedad de sentido del mundo y de su explotación, antes que solo de características del texto de la ley. ${ }^{19}$

Pese a que, por cierto, en los delitos de corrupción se manifiestan otros problemas jurídicos interesantes, lo que aquí me interesa hacer es leer el contenido y la evolución de los delitos de corrupción - y de ciertas prácticas de regulación y persecución - como reacciones frente

\footnotetext{
${ }^{17}$ En el caso de la corrupción política, la ambigüedad también es aumentada a causa de un fenómeno propio: la denuncia de corrupción es en sí un instrumento político y por ello un objeto de disputa en su sentido. Los políticos de derecha tienen interés en denunciar corrupción de sus rivales no solo por el provecho inmediato que supone la exclusión, sino también como modo de confirmación ideológica: el clientelismo y la ineficacia del Estado se demostrarían ante todo en la corrupción. Exactamente lo mismo tiene lugar en la izquierda: la corrupción demostraría que la derecha no es más que la representante de los intereses de las grandes empresas. LEVI (1997), pp. 35-48; NELKEN y LEVI (1996), pp. 1-17.

${ }^{18}$ ROSENBLATT (2012), pp. 237-51.

${ }^{19}$ Así también BLUNDO (2007), pp. 27-52.
} 


\section{Polít. Crim. Vol. 15, № 29 (Julio 2020), Art. 11, pp. 290-310 [http://politcrim.com/wp-content/uploads/2020/07/Vol15N29A11.pdf]}

a la resistencia natural que se presenta en este ámbito. La tesis es así sencilla: en tanto programa de resistencia al desarrollo de acciones corruptas de alto nivel, el derecho "en acción" con toda probabilidad se va a enfrentar frecuentemente a resistencia. Al hacer uso de programas de control que se fijan en una reconstrucción retrospectiva del sentido del comportamiento, como típicamente tiene lugar en el Derecho Penal, esa resistencia por explotación de ambigüedad y de sofisticación tiene un poder especialmente intenso ${ }^{20}$. Con ello, el derecho tiene que generar programas de reacción y esos programas de reacción han pasado a redefinir, con cierta claridad en Chile desde el caso Penta y la aprobación de la Ley 21.121, el Derecho Penal de la corrupción.

Los programas regulativos de reacción frente a la resistencia que me interesa analizar son tres.

El primero de ellos opera "en los libros", esto es, se trata de una técnica legislativa-formal de reacción frente a los problemas de ambigüedad que plantea la corrupción. Aquí, se trata de generar regulación determinada y resistente frente a la ambigüedad por la vía de romper la accesoriedad con la cultura que caracteriza a ciertas formas de regulación de la economía ordinaria. A este modo de regulación le llamo regulación por vía de tabuización de ciertas acciones.

Un segundo programa de resistencia no opera ya en los libros, sino que en "el derecho en acción”. La capacidad de explotación de la ambigüedad en ciertas clases de delitos puede tender a generar el efecto de que los encargados de hacer aplicar el Derecho Penal típicamente fiscales - dejen de perseguir por el título culturalmente relevante y, en cambio, generen prácticas oportunistas de persecución. A esto le llamo regulación por persecución oblicua (pretextual prosecution).

Finalmente, un último programa que opera más bien por intersección del derecho en los libros con el derecho en acción puede ser denominado regulación por vía de indeterminación. Aquí, los agentes encargados de aplicar y hacer aplicar la regulación hacen uso de regulación indeterminada para superar el efecto de la resistencia.

En lo que sigue, me interesa analizar progresivamente estos tres programas, dando cuenta de su racionalidad al hacer frente a la resistencia y de los problemas y riesgos que ellos presentan.

\section{Solución por vía de tabuización: la corrupción mínimamente accesoria}

\subsection{La técnica de la tabuización}

Como hemos visto, parte importante de los problemas que aquejan a la regulación de la corrupción de alto nivel se originan en la fuerte accesoriedad que puede tener el derecho como práctica social con las definiciones dominantes de la cultura. Ello entrega amplios espacios de explotación, intencional o no, a quien puede moverse con soltura en el mundo de

\footnotetext{
${ }^{20}$ Una pregunta distinta es, por cierto, en qué medida otros modos de regulación jurídica son equivalentes funcionales mejores. Entre nosotros véase a este respecto LONDOÑO (2014), pp. 147-67.
} 
la cultura dominante. Un modo de romper con esto consiste en privar de ambigüedad a ciertas prácticas: establecer el carácter ilícito sin más de conductas recurrentes en un ámbito determinado.

Esta técnica es fácilmente reconocible en la regulación del cohecho y del soborno público. Los delitos de cohecho ${ }^{21}$ se caracterizan por hacer punible, con relativa (o completa), simetría, la oferta de y la entrega de beneficios para la realización de una acción de servicio por parte de un funcionario público (soborno) y la solicitud de la entrega de un beneficio o su aceptación efectuada por un funcionario público a cambio de la ejecución de un acto de servicio (cohecho), como un modo de inmunizar al contexto de acción "administración" de la inserción de la lógica de intercambios privados.

El establecimiento histórico del cohecho se encuentra de hecho vinculado a esa posición: la regulación del cohecho es coincidente con la pretensión de establecimiento de una burocracia con un ethos en el que la realización de la función tuviera lugar en base a códigos distintos del comercio pero también de los intercambios de favores y regalos, presentes en otros ámbitos de la vida social. ${ }^{22}$ En qué medida esta representación sigue siendo relevante hoy, es algo que no tiene importancia aquí. Lo relevante es notar lo que supuso la coincidencia de la estructura del cohecho y la racionalidad burocrática: la pretensión irrestricta de separación del beneficio personal y el cumplimiento del cargo llevó, en los hechos, a una ruptura de la accesoriedad cultural que caracteriza en general a nuestra comprensión de la corrupción en aquello que entre dentro del ámbito del cohecho.

Así, la existencia de los delitos de cohecho implica que con completa independencia del perjuicio social o individual que suponga el intercambio en base al cual se realiza un acto de servicio, el ejercicio de una función pública no puede verse mediado por aceptaciones de beneficios externos al servicio. El establecimiento de esa prohibición y, con ello, la estabilización de la institución "burocracia" o "administración pública" a partir de ese código es el único sentido del cohecho. Todos esos conceptos no hacen más que dar cuenta de un hecho sencillo: el cohecho define negativamente a la institución administración pública y, por ello, no es más que parte de la institución misma. ${ }^{23}$

Dogmáticamente visto, ello hace que la discusión sobre el "bien jurídico afectado por los delitos de cohecho" 24 no tenga mayor relevancia. Distinguir si se trata de la protección de la "decencia (Lauterkeit) del ejercicio del cargo público", ${ }^{25}$ de la capacidad de funcionamiento de la administración del Estado, ${ }^{26}$ o de la confianza de la ciudadanía en las instituciones

\footnotetext{
${ }^{21}$ Sobre el concepto, véase solo OLIVER (2004), pp. 85-88.

${ }^{22}$ Sobre el vínculo con la representación de la burocracia prusiana en Alemania véase DURYNEK (2008), pp. 9 y ss.; ADAMSKI (2013), pp. 25 y ss. En el caso norteamericano y su pretensión de ruptura frente a la captura de las cortes europeas por los códigos de amabilidad personal e intereses particulares, véase TEACHOUT (2014), pp. 18 y ss.

${ }^{23} \mathrm{El}$ punto es tematizado correctamente ya por LOOS (1974), pp. 888 y ss. por referencia a la sociología de las instituciones de Schelsky.

${ }^{24}$ En general BANNENBERG (2002), pp. 14 y ss.; LOOS (1974), pp. 879 y ss.; HÖLTKEMEIER (2005), pp. 52ss.; MÖHRENSCHLAGER (2007), pp. 387-56, Nm. 6 y ss. Entre nosotros GARCÍA (2019), pp. 125-130; CALDERÓN (2004), pp. 93 y ss.

${ }^{25}$ Véase ROXIN (2015), p. 463.

${ }^{26}$ Así, por ejemplo, SIRCH (2008), pp. 57 y ss.
} 


\section{Polít. Crim. Vol. 15, № 29 (Julio 2020), Art. 11, pp. 290-310 [http://politcrim.com/wp-content/uploads/2020/07/Vol15N29A11.pdf]}

públicas, ${ }^{27}$ no es algo que tenga mayores efectos. Como institución, la administración pública depende del mantenimiento de un modo de acción y es obvio que el reconocimiento de una institución así reconocida depende de que el código en cuestión sea mantenido.

Pese a que el cohecho no sea más que parte de la definición de la institución administración pública, en el ámbito de los delitos de corrupción este puede ser estudiado como técnica de tabuización: ${ }^{28}$ la interpretación de la mera asunción de un rol determinado en un intercambio social implica prohibición de cualquier intercambio beneficioso porque la institución que se ve representada en ese rol supone independencia de la motivación que puede suponer la realización de la acción prohibida ${ }^{29}$ - el Estado es Estado de Derecho, eso quiere decir, acción motivada exclusivamente por aplicación de reglas - y porque ello permite limitar el riesgo de utilización de una posición de poder para puro beneficio personal. Al hacer esto, todo caso de cohecho pasa a poder ser interpretado como abuso o como modo de motivación incompatible.

La utilización de la técnica de la tabuización requiere así de la conjunción de dos elementos: fijación de un ámbito de acción suficientemente preciso e imposición de prohibiciones de contenido determinado en ese ámbito de acción. En el caso del cohecho, esa fijación de un ámbito de acción determinado es realizada por medio del concepto de empleado público y del concepto de acto del cargo (o por algún equivalente). ${ }^{30}$ Ambos conceptos son accesorios, en el sentido de que su contenido depende al menos parcialmente de regulación administrativa. Lo que, sin embargo, no es accesorio es la definición de las acciones prohibidas: la pura oferta/entrega/solicitud/aceptación de beneficios resulta en sí ilícita, con completa independencia de sus consecuencias o del grado de aceptación social que tenga. Con ello, se pretende generar algún grado de inmunidad frente a la resistencia y a la explotación de la ambigüedad.

La técnica de regulación por vía de tabuización tiene, por cierto, límites en la pretensión de regulación de la corrupción y en general de las acciones desviadas en la economía ordinaria. Parte de esos límites se juegan (3.2) en la interacción entre una técnica regulativa insuficientemente consciente de los problemas que tematiza y la interpretación de la regulación. Ella es así contingente. Pero los límites pueden derivar a su vez (3.3) de nada más que las limitaciones inherentes a la técnica en cuestión. En ambos casos, la tabuización muestra sus limitaciones frente al fenómeno de la resistencia.

\subsection{Insuficiencia de la tabuización por defectos de diseño}

\footnotetext{
${ }^{27}$ BGH en NStZ 1989, 76.

${ }^{28}$ Esto tiende a ser desconocido cuando se insiste en la inserción de tests de control de la afectación de "funciones específicas" o de "garantías de los ciudadanos". Así, por ejemplo, GARCÍA (2009), pp. 127-29; OLIVER (2004), pp. 94-95. El argumento retórico presentado a este respecto, es que de otro modo o bien "se moraliza la función pública" o "se asume una concepción autoritaria de las normas". Pero la cuestión es más sencilla: el cohecho participa de una práctica de exclusión de un tipo de acciones en un ámbito determinado. Es, por cierto, parte de un diseño institucional.

${ }^{29}$ Similar, en la orientación a la fijación de un cierto código de "imparcialidad", ARTAZA (2016), p. 322; MAÑALICH (2018), pp. 96-97.

${ }^{30}$ Similar GARCÍA (2019), passim, aunque insistiendo en la idea de intercambio ilícito como otro pilar central del sistema.
} 
El éxito de la técnica de la tabuización depende de su capacidad de excluir del ámbito de lo culturalmente aceptado las formas de interacción de que se trata. Como la función pretende ser realizada por medio de regulación formal, es probable que frente a ella se generen modos de elusión que tienen que ser enfrentados. Con ello, la verificación de técnicas de elusión aceptadas pasa a demostrar defectos de diseño que deben ser corregidos.

Un buen ejemplo de este fenómeno puede verificarse en la exclusión del soborno/cohecho por razón del cargo que tenía lugar en el derecho chileno con anterioridad a la promulgación de la Ley 21.121 el 20 de noviembre de $2018^{31}$. Antes de la modificación, la realización del delito de cohecho era dependiente de la posibilidad de verificar un intercambio injusto preciso entre un acto del cargo y la entrega o aceptación de entrega de beneficios. Ello pasó a generar dos problemas recurrentes.

El primer problema era de pura eficacia por dificultad probatoria: no siempre es sencillo probar que un empleado público solicitó para ejecutar una acción precisa del cargo ${ }^{32}$. Ello puede, además, ser internalizado por la práctica: para sobornar o solicitar coimas, es conveniente como estrategia de elusión no solo intentar esconder que se recibió un beneficio, sino esconder el acto que se ejecutó en beneficio del sobornante en un gran número de actos que lleven naturalmente a la realización de ese acto.

El segundo problema es sustantivo, relativo a la tipicidad de una forma de relación con la administración pública: el modelo tradicional del cohecho hace que, en general, la pura compra/venta de la lealtad del empleado público con un agente económico no sea punible y que incluso el favorecimiento posterior no lo sea $^{33}$, si es que la acción específica ejecutada en beneficio del externo no había sido "subjetivamente" determinada (aunque sea a grandes rasgos) al momento del intercambio ${ }^{34}$. En los hechos, esto es, sin embargo, recurrente en la fenomenología de la corrupción. El iter normal del desarrollo de relaciones corruptas (sean sectoriales, relativas a una relación precisa o incluso sistémicas) tiende a comenzar con iniciativas codificadas como altruistas en la generación de una "relación de confianza" 35 , para luego recibir regalos y otros beneficios como parte de ese buen clima que existe entre las

\footnotetext{
${ }^{31}$ Notablemente, un antecedente importante utilizado en el contexto de tramitación de esta ley, para generar convicción sobre la necesidad de criminalizar el cohecho en razón del cargo, fue una minuta confeccionada por el profesor Héctor Hernández que advertía precisamente el defecto de diseño. Ella puede ser consultada en http://www.senado.cl/appsenado/index.php?mo=tramitacion\&ac=getDocto\&iddocto=3017\&tipodoc=docto c omision. Posteriormente, fue publicada en HERNÁNDEZ (2016), pp. 15-28.

${ }^{32}$ Así también HERNÁNDEZ (2016), passim.

${ }^{33}$ Sobre el así denominado "cohecho subsiguiente", véase solo OLIVER (2004), p. 88.

${ }^{34}$ Por supuesto - y esto tiene relevancia dogmática en Chile — eso no significa que la determinación de la acción del cargo por la que se intercambia el beneficio haya debido ser determinada hasta en sus detalles o incluso individualizada. En Alemania, el BGH ya había resuelto antes de la reforma que la acción solo debía referirse a actividades determinables; que fuera claro que se esperaba realizara "algo" a cambio del beneficio entregado (BGHSt 32, 290; 39, 45). La diferencia después de la modificación es que solo es necesario construir una expectativa cuyo contenido puede no ser determinable al momento de la entrega del beneficio. En ese sentido también BGHSt 53, 6. La pretensión central es, con ello, cubrir una mayor parte del iter común de la corrupción. Sobre este punto, véase también MAÑALCH (2018), pp. 113-16.

${ }^{35}$ En Alemania, el concepto informal que tiende a ser utilizado para referirse al tipo de prácticas que quedaban excluidas con la regulación del cohecho antes de la reforma de 1997 era Anfütterung (el concepto proviene de la pesca y hace referencia a la utilización del cebo para atraer uno o más peces) o la expresión eufemística sacada de la propia práctica Klimapflege (cuidado del ambiente de trabajo entre el privado y la administración).
} 


\section{Polít. Crim. Vol. 15, No 29 (Julio 2020), Art. 11, pp. 290-310 [http://politcrim.com/wp-content/uploads/2020/07/Vol15N29A11.pdf]}

partes y solo cuando la relación es segura, al ya estar mediada por una lealtad adquirida entre otras cosas a base de la entrega de beneficio, se realiza el encargo ${ }^{36}$. El caso es menos problemático cuando surge desde el empleado público, ya que, pese a la generación de confianza mutua en base a intercambios de beneficios anteriores, el empleado no va a tender a ofrecer realizar un acto del cargo sino a cambio de un beneficio posterior ${ }^{37}$.

La persistencia de estos problemas en la práctica chilena muestra que, juzgada desde el punto de vista del puro cumplimiento de la función de tabuización del tipo de intercambios que quieren ser excluidos, el derecho chileno presentaba deficiencias. La Ley 21.121 puede ser leída como corrección de esos defectos y, así, como una forma de reacción frente a las estrategias de resistencia que se habían presentado.

\subsection{El efecto de retroalimentación limitante de la técnica de la tabuización}

En segundo lugar, la construcción de categorías por estructuras rígidas, ligadas a la idea de regulación por tabuización, puede producir problemas cuando esa estructura genera un efecto de retroalimentación en la categoría normativa que pretendía ilustrar. Como cuestión social, la técnica de la tabuización trae el riesgo de servir para aumentar el efecto de la sofisticación por simple diferenciación de la conducta con la estructura rígida.

Aunque esta idea de retroalimentación puede parecer compleja, en realidad ella es sencilla y fácilmente observable. El ejemplo más relevante para ilustrar esta idea puede encontrarse en la construcción del concepto de "corrupción política". Si el concepto de corrupción es construido sobre la base del intercambio injusto (Unrechtsvereinbarung, quid pro quo) que caracteriza a los delitos de cohecho, entonces todo comportamiento que no se estructure sobre un intercambio de esta clase no podría ser constitutivo de corrupción una vez generado el efecto de retroalimentación. La corrupción política, por ejemplo, pasaría a estar definida en relación a la entrega de ventajas a cambio de un acto del cargo, excluyendo de entrada como caso de corrupción política a aquellos supuestos de hecho que no satisfagan esta descripción. Puede parecer sencillo replicar a esto que no es problemático distinguir entre corrupción como concepto dogmático-penal y corrupción como concepto general; que no hay problemas con considerar que hay corrupción política, por ejemplo, en el financiamiento ilegal constante de ciertos candidatos o partidos pese a que no pueda comprobarse que haya corrupción en sentido jurídico-penal. Pero es probable que la utilización jurídico-penal excluyente del concepto de corrupción sea aprovechada para excluir casos que identificamos claramente en el plano de la cultura como "corruptos". Esta alegación es, de hecho, constante: aquello que hizo un político, un empresario u otro agente no sería punible (y antes de eso: se presumiría inocencia), por lo que no sería corrupción en el sentido relevante de la palabra, aunque podría haber algún problema ético.

\footnotetext{
${ }^{36}$ Sobre el iter del desarrollo fenoménico de relaciones corruptas, véase BANNENBERG (2002), pp. 103 y ss.

${ }^{37}$ En Alemania, la solución de estos problemas no implicó, como a veces se cree, la erradicación de toda exigencia de intercambio, sino lo que es típicamente denominada como una "relajación" (Lockerung) en su interpretación: el intercambio ya no se define en base a una acción precisa del cargo, sino a expectativas reconocibles de favorecimiento que pueden no estar determinadas. Sobre la modificación legal respectiva véase BANNENBERG (2002), pp. 17 y ss.; DURYNEK (2008), pp. 46 y ss.; SIRCH (2008), pp. 102 y ss.; MÖHRENSCHLAGER (2007), Nm. 62s.; KNAUER y KASPAR (2005), p. 393. En Suiza PIETH (2016), pp. 180 y ss.
} 
El problema no es a este respecto "puramente comunicacional". ${ }^{38}$ Ello se demuestra dramáticamente en la evolución de la regulación constitucional de las leyes de regulación de las campañas políticas en los Estados Unidos. Allí, a causa de la asunción de que el concepto de corrupción necesita de un quid pro quo específico, la Corte Suprema Federal pudo llegar a la conclusión de que la imposición de límites a la capacidad de participación de grandes patrimonios en financiamiento de campañas políticas y la limitación de las corporaciones como donantes serían contrarios a la Primera Enmienda.

La historia es conocida: ${ }^{39}$ en Buckley v. Valeo, ${ }^{40}$ la Corte Suprema Federal invalidó en el año 1977 parte de la Ley de Campañas Electorales Federales ("FECA") en lo que se refería a los límites en los gastos de campaña impuestos por ella y mantuvo, en cambio, parte de las limitaciones relativas a la magnitud de las donaciones. La diferencia fue justificada en relación a que en el ámbito de la regulación de las donaciones habría un interés constitucionalmente relevante en evitar corrupción. Esto no es en sí problemático. Lo que devino problemático con el tiempo es el concepto de corrupción que utilizó: corrupción, en su comprensión, sería la obtención de actos de servicio del candidato una vez electo a cambio de (grandes) donaciones de dinero. ${ }^{41}$

Aunque puede argumentarse que Buckley fue posteriormente deformado por la línea jurisprudencial que nos interesa, la sentencia permitió reducir la regulación constitucionalmente permitida de los modos de financiamiento y gasto en campañas a aquello que implicara un riesgo de corrupción definido estrechamente por referencia al cohecho (bribery). La corrupción política no se vería así constituida por creaciones de comunidades de intereses entre donantes y políticos o por el exceso de influencia y de poder de defensa de intereses particulares que, en general, adquirirían los grandes donantes, sino por aparición o riesgo de aparición de intercambios precisos injustos.

Ello llegó a su paroxismo en Citizens United v. FEC ${ }^{42}$ y McCutcheon v. FEC: ${ }^{43}$ por exigencias de la Primera Enmienda, y a falta de corrupción en el sentido relevante de la palabra, no podría regularse restrictivamente la capacidad de donación de personas jurídicas ni podrían imponerse límites agregados en las donaciones realizadas por individuos determinados.

Así, la representación de que el concepto institucionalmente relevante de corrupción se reduce a la estructura del cohecho llevó a excluir de posibilidad de regulación aquello que no se vinculara directamente con eliminación del cohecho, pese a la obviedad del exceso de influencia que los grandes donantes han asumido en la política federal estadounidense.

\footnotetext{
${ }^{38}$ Así TEACHOUT (2014), pp. 5 y ss.

${ }^{39}$ En esto me baso ante todo en el lúcido análisis de TEACHOUT (2014), pp. 6 y ss., pp. 276 y ss.

40424 U.S. 1 (1976).

${ }^{41}$ Véase LOWENSTEIN (1989), pp. 29 y ss. El artículo de Lowenstein contiene una crítica general al modo de razonar jurídico en la asunción de que la relativa claridad de nuestra comprensión del cohecho (bribery) permitiría definir de mejor modo la relativamente indeterminada corrupción.

42558 U.S. 310 (2010).

43572 U.S. 185 (2014).
} 


\section{Polít. Crim. Vol. 15, No 29 (Julio 2020), Art. 11, pp. 290-310 [http://politcrim.com/wp-content/uploads/2020/07/Vol15N29A11.pdf]}

Aquí no puedo profundizar demasiado sobre las razones que hacen posible la generación de este efecto de retroalimentación. Es probable que el exceso de atención a categorías morales de incorrección (moral wrongdoing) en ámbitos regulatorios, saturados de juegos de explotación de ambigüedad, contribuya a ello. Para nuestros efectos, el estudio de las causas es menos relevantes que la pura verificación de los límites de la regulación por vía de tabuización: dadas sus limitaciones naturales, y la oferta de explotación que ella supone, es probable que agentes de control cuya función se vea definida en relación con el combate de la corrupción (periodistas especializados, fiscales anti-corrupción, grupos políticos anticorrupción) asuman que otras formas de reacción son necesarias.

\section{La evolución: Persecución indirecta e indeterminación como técnicas de regulación}

Las limitaciones, tanto inmanentes como contingentes, de la técnica de la ruptura de la accesoriedad cultural por la vía de la tabuización de intercambios determinados sugieren que, si bien ella puede cumplir un rol importante, resulta insuficiente como programa de regulación excluyente.

Las limitaciones de técnicas con este grado de determinación generan, en lo que alcanzo a reconocer, dos vías por las cuales los sistemas de persecución penal intentan superarlas. El ejercicio que hemos venido desarrollando insinúa que probablemente el verdadero problema al que debe enfrentarse la academia es al análisis normativo de estos dos modos de acción.

\subsection{La persecución oblicua y sus riesgos}

El primero puede llamarse persecución oblicua (pretextual prosecution). ${ }^{44} \mathrm{Su}$ uso es fácilmente reconocible en la experiencia chilena y comparada en distintos contextos. Asumamos que la posibilidad del uso de potestades estatales para provecho privado se encuentra en el origen de la necesidad regulativa que dio origen a los delitos formales de corrupción como delitos de cohecho. ${ }^{45}$ Esa misma pretensión se juega, sin embargo, en el tipo de casos que en Chile se han conocido como "casos de corrupción" pero que en la mayor parte de ellos no son constitutivos de (o al menos no terminan siendo tratados como) soborno/cohecho. En ellos, partidos políticos o candidatos eran financiados fuera de los mecanismos permitidos por la ley por grupos empresariales y emitían boletas de prestación de servicio directamente o por intermedio de un tercero. Aquello que como fenómeno caracteriza a la corrupción se encuentra sin dudas presente aquí, pero a falta de un intercambio preciso del tipo que exigía el cohecho en el derecho positivo chileno - un beneficio innominado a cambio de una acción del cargo- - el sistema no tendía a aceptar su procesamiento como delito de cohecho. Pese a ello, se sigue tratando del tipo de situaciones que interesa regular al hablar de corrupción.

En contextos de esta clase, no es inusual que se generen estrategias de persecución oblicua. En los casos de persecución oblicua, los persecutores se concentran en determinados individuos, a quienes pretenden someter a procesamiento y castigo penal por un título

\footnotetext{
${ }^{44} \mathrm{Al}$ respecto, RICHMAN y STUNTZ (2005), pp. 583-640.

${ }^{45}$ Al respecto véase, por ejemplo, DURYNEK (2008), pp. 9 y ss.; ADAMSKI (2013), pp. 25 y ss.
} 
determinado pero que se asume como débil en el foro. ${ }^{46}$ Por la dispersión normativa que caracteriza a las sociedades modernas, en estos casos no es difícil encontrarse con otros títulos que sí pueden tener mayores posibilidades de éxito. En los Estados Unidos, el fraude postal o el perjurio en documentos oficiales ${ }^{47}$ - entre otros - tienden a ser utilizados como tipos que permiten cumplir esta función en el sistema federal. En Chile, la función era probablemente cumplida ante todo por delitos tributarios y por delitos de falsedad vinculados a los mismos documentos tributarios. La estrategia ha tendido a ser menos exitosa, por la sencilla razón de que el ejercicio de acciones penales en este ámbito depende del Servicio de Impuestos Internos. Al ser menos probable que allí se generen identidades que motiven a la persecución personal, ello limita la extensión y la eficacia de la persecución oblicua.

La persecución oblicua plantea problemas normativos tanto a nivel político como a nivel jurídico. ${ }^{48}$ En el ambiente académico, pero centrado más bien a nivel político, los problemas pueden ser procesados (o problematizados) por medio de la exigencia más amplia de fair labeling en la criminalización ${ }^{49} \mathrm{o}$, en general de imparcialidad y justicia (fairness) en la persecución. ${ }^{50}$ Un sistema, como el sistema federal norteamericano, ${ }^{51}$ ampliamente orientado a la persecución oblicua, ${ }^{52}$ perdería la orientación general a la aplicación imparcial a la ley $\mathrm{y}$, en algunos casos, podría generar prácticas especialmente problemáticas ${ }^{53}$ (ej.: perseguir por medio de la aplicación de leyes en desuso). Más convincente, el sistema podría perder eficacia comunicativa en combatir lo que pretende: al concentrarse en personas antes que en clases de acciones, el reforzamiento normativo de lo que verdaderamente se pretende combatir (aquí: corrupción) sería menos eficiente. ${ }^{54}$

Para nuestros efectos, solo es relevante reconocer la dinámica de tensiones producidas por estructura misma de la corrupción de alto nivel — y, más generalmente, de la delincuencia de alto nivel: las dificultades ligadas a la resistencia formal generan incentivos a la expansión de las posibles fuentes de acusación para superarla. Ella genera, entre otras cosas, prácticas de persecución oblicua como mecanismo tendiente a contrarrestar la resistencia. Dada la naturaleza del conflicto en cuestión, simplemente descartar el fenómeno como desviación tiene poco rendimiento analítico y, además, probablemente resulte poco eficiente como solución que un sistema legal pueda aceptar. En sociedades complejas, se requiere antes un análisis más fino del fenómeno de la persecución oblicua.

\footnotetext{
46 Sobre la causa del uso de técnicas de persecución oblicuas, véase RICHMAN y STUNTZ (2005), pp. 588-591.

${ }^{47}$ RICHMAN y STUNTZ (2005), p. 584.

48 Véase LITMAN (2004), pp. 1149 y ss.

${ }^{49}$ El concepto proviene del clásico trabajo de WILLIAMS (1983), pp. 85-95. Sobre la discusión, más recientemente CHALMERS y LEVERICK (2008), pp. 217-46.

${ }^{50}$ Esta es la etiqueta general con la que se discute en los Estados Unidos. Véase LITMAN (2004), pp. 1149 y ss.

51 Véase, sin embargo, SKLANSKY (2018), pp. 454-455; SKLANSKY (2016), pp. 485-487., notando el aumento de la relevancia de esta orientación en contextos estatales de persecución.

52 RICHMAN y STUNTZ (2005), pp. 608-614.

${ }^{53}$ Así, por ejemplo, SKLANSKY (2016), pp. 508 y ss.: el problema central de la persecución oblicua es que expresa una comprensión puramente instrumental de la ley, en la que lo que importa es la probabilidad de obtener resultados valorados con independencia de ella.

${ }^{54}$ Este es el argumento central de RICHMAN y STUNTZ (2005), passim.
} 


\section{Polít. Crim. Vol. 15, No 29 (Julio 2020), Art. 11, pp. 290-310 [http://politcrim.com/wp-content/uploads/2020/07/Vol15N29A11.pdf]}

\subsection{La inversión del problema original: regulación por uso de la indeterminación}

La persecución oblicua no es el único programa regulativo observable a este respecto. Un segundo programa puede denominarse "regulación por vía de indeterminación": generar reglas cuya dificultad de determinación fija del sentido permitan evitar los efectos de conductas elusivas. En Alemania, una discusión de esta clase se ha producido a propósito de la función que ha pasado a cumplir el tipo de administración desleal desde los escándalos de corrupción de los años 90s y 2000s.

El ejemplo más obvio de un caso fenoménicamente calificable como "de corrupción” pero al que solo son aplicables otras clases de delitos es la denominada "formación de cajas negras" 55 - algo que conocemos en Chile, aunque no parece haber sido discutido del todo, en el caso Soquimich. ${ }^{56}$ En los casos de cajas negras, un funcionario o una división dentro de una empresa separan ciertos fondos de la contabilidad general de la empresa y los ponen a disposición inmediata y discrecional de ciertos agentes ("caja"), con el objeto de realizar pagos que por distintos motivos (ilegalidad, conflicto interno a la empresa) quieran ocultarse a externos. Sea por inexistencia de un delito de corrupción entre privados cuando las coimas se pagan a propósito de la contratación privada, sea porque las coimas no se pagaron o no se puede probar qué coimas se pagaron en el sector público, o porque ello tuvo lugar en un contexto que no es punible conforme al derecho nacional - esto sucede en varias legislaciones con los denominados facilitation payments ${ }^{57}$-, el caso puede no ser punible conforme a los delitos formales de corrupción. La formación misma de la caja negra puede ser, sin embargo, punible a otro título (administración desleal, alguna clase de falsedad contable). ${ }^{58}$

Aquí, el caso es ciertamente tratado como de corrupción por asimilación, si se quiere, parasitaria: porque la caja se forma para pagar coimas, no tenemos problemas con identificarlo como un caso de corrupción. Lo mismo sucede en el caso de la persecución del pago de una cantidad de dinero contra informes falsos: porque hay un intercambio ilícito pero

\footnotetext{
${ }^{55}$ MÖHRENSCHLAGER (2007), pp. 122 y ss.; BERNSMANN y GATZWEILER (2014), Nm. 693 y ss.; TSAGKARAKI (2013), pp. 23 y ss.; FISCHER (2010), pp. 15 y ss. Sobre el caso Siemens y la evolución jurisprudencial que produjo véase GRAF (2016), pp. 7 y ss.

${ }^{56}$ No es casual que las razones de formación de algo así como una caja negra (aquí: puesta a disposición del Gerente General de Soquimich, Patricio Contesse, de fondos que eran manejados discrecionalmente y sin entrega de información por él) tuviera más o menos la misma función que tiende a presentar en el derecho comparado, a saber, que sirva a realizar pagos que se pretenden mantener escondidos (coimas, financiamiento político). Sobre las motivaciones en Alemania, véase TSAGKARAKI (2013), pp. 48 y ss.

${ }^{57} \mathrm{Al}$ respecto véase solo DORSCHFELDT (2016), pp. 41 y ss.

${ }^{58}$ En Alemania, la discusión se centra en la pregunta por si el mero hecho de la formación de una caja negra, a causa de la privación del uso de los fondos puestos en ella, implica perjuicio y, de este modo, realización del delito de administración desleal, con independencia de que los fondos en cuestión sean (como en general pero no siempre lo son) utilizados para satisfacer fines sociales. Este es el modo en que la cuestión es tratada hasta el día de hoy por la jurisprudencia. Véase RGSt 71, 155; 157, 73; BGHSt 19, 37; 40, 287 (todos ellos en el ámbito particular de lo que entre nosotros sería malversación de fondos públicos). En el sector privado fundamental BGHSt 52, 323; 55, 266. La alternativa consiste en vincular la punibilidad a la irrogación de alguna clase de perjuicio económico, incluyendo el denominado "perjuicio por riesgo" a partir de la generación del peligro de sanción. Ello supone, sin embargo, ilicitud de la conducta en base a normas de derecho público y no pura infracción de reglas societarias.
} 
que no es punible en sí, el caso es construido como de corrupción, pese a que no se apliquen tipos de cohecho sino principalmente delitos tributarios. ${ }^{59}$

Pero el fenómeno de la asimilación parasitaria muestra que el control estatal puede no seguir las lógicas formales del derecho sin por ello incurrir en errores en la aplicación de la ley: el caso es perseguido como de corrupción y por tratarse de corrupción pese a que no haya cohecho punible. Mejor: la aplicabilidad formal de otros títulos de punibilidad es utilizada para satisfacer expectativas de persecución ligadas a prácticas que son vistas como corruptas.

Aquí solo me interesa notar que, frente al programa de entrega de municiones a la persecución oblicua, el programa de generación de tipos amplios e indeterminados genera no solo problemas políticos o de regulación de discreción administrativa, sino propiamente dogmáticos. Es obvio que frente a la pura existencia de este programa, los penalistas inmediatamente detectarían que hay un problema en relación con el principio de legalidad en su vertiente de lex certa. Puede ser. Pero uno se adelanta y no entiende correctamente el problema si no se fija en el contexto en el que tiene lugar este uso de la indeterminación: responde ante todo a la impotencia del derecho y de sus agentes frente a la evidencia de la explotación de su propia indeterminación. ${ }^{60}$

\section{Conclusiones}

El derecho tiene una capacidad limitada de acción como mecanismo regulatorio y de control del mundo ordinario. Esas limitaciones se explican, al menos en parte, por la mucho mayor resistencia que los sujetos que se desempeñan en ese mundo, y las acciones que se desarrollan en él, tienen frente a su consideración como desviados, ilícitos, etc. Enfrentados frente a pretensión de regulación de esta clase, los sujetos y sus acciones manifiestan resistenciaalgo que naturalmente denota privilegio, captura del Estado y de la ley, o al menos ineficacia de uno y otro.

Sería un error, sin embargo, creer que los agentes del sistema de justicia - y en menor medida del sistema político - no reaccionan frente a esta realidad. Al contrario, el artículo identifica tres formas de reacción, cuyo objeto es evitar la resistencia frente a la regulación que se manifiesta en general en la economía ordinaria y especialmente en la corrupción de alto nivel. La primera forma consiste en tabuizar un modo de interacción en ciertos contextos de interacción. La exclusión de la toma de beneficios personales ajenos al cargo, por ejemplo, está directamente vinculada al cohecho en el sector público. Pero en tanto modelo regulatorio formal, éste se encuentra también abierto a elusión y resistencia-una realidad probablemente resentida por los agentes de control. En ese contexto, el "derecho en acción"

\footnotetext{
${ }^{59}$ De nuevo pese a que el modus operandi en cuestión nos pueda parecer idiosincráticamente natural, se trata de un modo de acción que ha sido internacionalmente relevante. En Alemania, por ejemplo, el pago por supuestos informes constituía uno de los modos en que más frecuentemente se financiaba ilegalmente a partidos políticos y candidatos durante el gran escándalo de "donaciones a partidos políticos" que se desarrolló entre los años 80 y no se cerró hasta mediados de los 2000. Al respecto, WOLFSLAST (1995), pp. 124 y ss.; SCHREIBER (1989), pp. 2 y ss.

${ }^{60}$ Enfático NAUCKE(2012), passim. Ello explica la apertura del BVerfG a su consideración como un modo de regulación válido, en la medida en que se vea controlado por una práctica judicial y doctrinal sólida. Véase BVerfGE 126, 170. Al respecto, con detalle GRAF (2016), passim.
} 
Polít. Crim. Vol. 15, No 29 (Julio 2020), Art. 11, pp. 290-310

[http://politcrim.com/wp-content/uploads/2020/07/Vol15N29A11.pdf]

nacional y comparado ha tendido a mostrar al menos dos formas adicionales de reacción frente a la resistencia: la generación de técnicas y orientaciones a la persecución oblicua, por una parte, y el uso de reglas con contenido indeterminado y programas progresivos de aplicación, por la otra.

Aunque el artículo presenta un breve análisis de los riesgos de estas dos formas de acción, su pretensión central es dar cuenta de su existencia, y del contexto en que se insertan, como modo de llamar a complementar las discusiones que se dan entre nosotros a este respecto. 
WILENMANN, Javier: "El derecho frente a la resistencia a la criminalización bajo el ejemplo de los delitos de corrupción".

\section{Bibliografía}

ADAMSKI, Vivien (2013): Die gesetzliche Konzeption der Bestechungsdelikte (Frankfurt am Main: Peter Lang).

ANAND, Vikas; ASHFORTH, Blake; JOSHI, Mahendra (2004): "Business as Usual: The Acceptance and Perception of Corruption in Organizations", en: The Academy of Management Executive (vol. 18 no. 2), pp. 39-53.

ARNOLD, Thurman (2010) The Folklore of Capitalism, nueva edición (New Brunswick/Londres: Transaction Publishers).

ARTAZA, Osvaldo (2016) "La utilidad del concepto de corrupción de cara a la delimitación de la conducta típica en el delito de cohecho", en: Politica Criminal (vol. 11 no. 21), pp. 307-39.

BANNENBERG, Britta (2002): Korruption in Deutschland und ihre strafrechtliche Kontrolle (Neuwied: Luchterhand).

BECKER, Howard (1963): Outsiders. Studies in the Sociology of Deviance (Nueva York: Free Press of Glencoe).

BERNSMANN, Klaus y GATZWEILER, Norbert (2014): Verteidigung bei Korruptionsfällen, 2. edición (Heidelberg: C.F. Müller).

BLUNDO, Giorgio (2007): "Hidden Acts, Open Talks. How Anthropology can 'Observe' and Describe Corruption", en: ANDERS y NUIJTEN (eds.), Corruption and the Secret of Law (Londres: Routledge), pp. 27-52.

CHALMERS, James y LEVERICK, Fiona (2008) "Fair Labelling in Criminal Law", en: The Modern Law Review (vol. 71), pp. 217-46.

COLEMAN, James William (1987): "Toward an Integrated Theory of White-Collar Crime", en: American Journal of Sociology (vol. 93 no. 2), pp. 406-39.

DE GRAAF, Gjalt (2007): "Causes of Corruption: Towards a Contextual Theory of Corruption” en: Public Administration Quarterly (vol. 31 no. 1/2), pp. 39-86.

DE GRAAF, Gjalt; HUBERTS, L. (2008): "Portraying the Nature of Corruption Using an Explorative Case Study Design”, en: Public Administration Review (vol. 68 no. 4), pp. 640-53.

DEN NIEUWENBOER, Neil; KAPTEIN, Muel (2008): "Spiraling Down into Corruption: A Dynamic Analysis of the Social Identity Processes That Cause Corruption to Grow in Organizations", en: Journal of Business Ethics (vol. 83 no. 2), pp.133-46.

DÖLLING, Dieter (2007): “Grundlagen der Korruptionsprävention”, en: DÖLLING, Dieter (ed.), Handbuch der Korruptionsprävention (Múnich: C.H. Beck), pp. 1-40.

DORSCHFELDT, Dorian (2016): Strafbarkeit von Facilitation Payments (Berlín: Berliner Wissenschafts-Verlag).

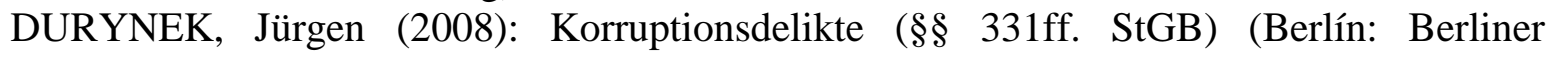
Wissenschafts-Verlag).

EARLE, John S.; SPICER, Andrew y PETER, Klara (2010): "The Normalization of Deviant Organizational Practices: Wage Arrears in Russia, 1991-98", en Academy of Management Journa (vol. 53 no. 2), pp. 218-37.

FISCHER, Thomas (2010): "Wirtschaftskriminalität und Korruption. Aktuelle Entwicklungen in der Rechtssprechung", en: BANNENBERG y JEHLE (eds.), Wirtschaftskriminalität (Mönchengladbach: Forum Verlag Godesberg), pp. 3-24.

GALPERIN, Bella L.; BENNETT, Rebecca J.; y AQUINO, Karl (2011): "Status Differentiation and the Protean Self: A Social-Cognitive Model of Unethical 
Behavior in Organizations", Journal of Business Ethic (vol. 98 no. 3), pp. 407-24.

GARCÍA, Gonzalo (2019): "(In)compatibilidad del financiamiento ilegal de la actividad parlamentaria con el delito de cohecho", en: Politica Criminal (vol. 14 no. 27), pp. $122-72$.

GRAF, Pia-Franziska (2016): Das Vermögensstrafrecht vor den Schranken des Verfassungsrechts (Marburg: Tectum Verlag).

GREEN, Stuart P. (2004): "The Concept of White Collar Crime in Law and Legal Theory", en: Buffalo Criminal Law Review (vol. 8 no. 1), pp. 1-34.

HARRIS, Robert (2003): Political Corruption. In and beyond the Nation State (Londres/Nueva York, Routledge).

HERNÁNDEZ, Héctor (2016): "La inconveniente exigencia de un acto funcionario determinado como contraprestación en el delito de cohecho", en: Revista de Ciencias Penales (vol. 43 no. 4), pp. 15-28.

HÖLTKEMEIER, Kai (2005): Sponsoring als Straftat (Berlín: Duncker \& Humblot).

KNAUER, Christoph; KASPAR, Johannes (2005): "Restriktives Normverständnis nach dem Korruptionsbekämpfungsgesetz”, en: Goltdammer's Archiv für Strafrecht, pp. 385405.

LEVI, Michael (1997): “The Crime of Corruption”, en RIDER, Barry (ed.), Corruption: The Enemy Within (The Hague: Kluwer Law International), pp. 35-48

LITMAN, Harry (2004): "Pretextual Prosecution", en: The Georgetown Law Journal (vol. 92), pp. 1135-80.

LONDOÑO, Fernando (2014): "Tipicidad y legalidad en el derecho administrativo sancionatorio", en: Revista de Derecho (Valdivia) (vol. 27 no. 2), pp. 147-67.

LOOS, Fritz (1974): "Zum 'Rechtsgut' der Bestechungsdelikte”, en: STRATENWERTH ET AL (eds.), Festschrift für Hans Welzel (Berlín/Nueva York: De Gruyter), pp. 87995.

LOWENSTEIN, Daniel (1989): "Legal Efforts to Define Political Bribery", en HEIDENHEIMER et al. (eds.), en: Political Corruption. A Handbook (New Brunswick: Transaction Publishers), pp. 29-38.

MAÑALICH, Juan Pablo (2018): "El cohecho como propuesta o aceptación de una donación remuneratoria", en: Revista de Derecho de la Pontificia Universidad Católica de Valparaíso (vol. 51), pp. 95-122.

MISANGYI, Vilmos; WEAVER, Gary y ELMS, Heather (2008): "Ending Corruption: The Interplay Among Institutional Logics, Resources and Institutional Entrepreneurs", en: The Academy of Management Review (vol. 33 no. 3), pp. 750-70.

MÖHRENSCHLAGER, Manfred (2007): “Der strafrechtliche Schutz gegen Korruption”, en DÖLLING (ed.) Handbuch der Korruptionsprävention (Múnich: C.H. Beck), pp. $387-456$.

NAUCKE, Wolfgang (2012): Der Begriff der politischen Wirtschaftsstraftat (Berlín: Lit Verlag).

NELKEN, David; LEVI, Michael (1996): "The Corruption of Politics and the Politics of Corruption: An Overview", en: Journal of Law and Society (vol. 23 no. 1), pp. 1-17.

NIELSEN, Richard (2003): "Corruption Networks and Implications for Ethical Corruption Reform", en: Journal of Business Ethics (vol. 42 no. 2), pp. 125-49.

NUIJTEN, Monique; ANDERS, Gerhard (2007): "Corruption and the Secret of Law: An Introduction”, en: NUIJTEN, Monique; ANDERS, Gerhard (eds.), Corruption and the Secret of Law (Farnham/Burlington: Ashgate Publishing), pp. 1-26. 
OLIVER, Guillermo (2004): “Aproximación al delito de cohecho”, en: Revista de Estudios de la Justicia (vol. 5), pp. 83-115.

PIETH, Mark (2016): Wirtschaftsstrafrecht (Basilea: Helbing Lichtenhan Verlag).

PINTO, Jonathan; LEANA, Carrie y PIL, Frits (2008): "Corrupt Organizations or Organizations of Corrupt Individuals? Two Types of Organizational-Level Corruption", en: Academy of Management Review (vol. 33 no. 3), pp. 685-709.

RICHMAN, Dan; STUNTZ, William (2005): "Al Capone's Revenge: An Essay of the Political Economy of Pretextual Prosecution”, en: Columbia Law Review (vol. 105 no. 2), pp. 583-640.

ROSENBLATT, Valerie (2012): "Hierarchies, Power Inequalities, and Organizational Corruption", en: Journal of Business Ethics (vol. 111 no. 2), pp. 237-51.

ROXIN, Claus (2015): "Vorteilsannahme", en ALBRECHT et al, Festschrift für Walter Kargl zum 70. Geburtstag (Berlín: Berliner Wissenschafts-Verlag), pp. 459-77.

SCHÖN, Felix (2011): Korruption (Frankfurt am Main: Verlag für Polizeiwissenschaft).

SCHREIBER, Hans-Ludwig (1989): Parteispenden dnd Strafrecht (Colonia: Verlag Josef Eul).

SHAPIRO, Susan (1990): "Collaring the Crime, Not the Criminal: Reconsidering the Concept of White-Collar Crime”, en: American Sociological Review (vol. 55 no. 3), pp. 346-65.

SIRCH, Christian (2008): Die Strafbarkeit der Parteispendenakquisition (Frankfurt am Main: Peter Lang).

SKLANSKY, David (2016): "The Nature and Function of Prosecutorial Power", en: The Journal of Criminal Law and Criminology (vol. 106 no. 3), pp. 473-520.

SKLANSKY, David (2008): "The Problems with Prosecutors", en: Annual Review of Criminology (vol. 1), pp. 451-69.

SUTHERLAND, Edwin (1945): "Is 'White Collar Crime' Crime?", en: American Sociological Review (vol. 10 no. 2), pp. 132-39.

SUTHERLAND, Edwin (1940): "White-Collar Criminality", en: American Sociological Review (vol. 5 no. 1), pp. 1-12.

SUTHERLAND, Edwin (1949): White Collar Crime (Nueva York: The Dryden Press).

TAVITS, Margit (2010): "Why Do People Engage in Corruption? The Case of Estonia", en: Social Forces (vol. 88 no. 3), pp. 1257-79.

TEACHOUT, Zephyr (2014): Corruption in America (Cambridge/Londres: Harvard University Press).

TIEDEMANN, Klaus (2014): "Die Gesetzumgehung Als Zentralthema Des Wirtschaftsstrafrechts Am Beispiel Des Ärtzlichen Abrechnungsbetruges”, en HEGER et al (eds.), Festschrift für Kristian Kühl zum 70. Geburtstag (Múnich: C.H. Beck), pp. 735-46.

TSAGKARAKI, Eirini (2013): Die Bildung der Sog. "schwarzen Kassen” als strafbare Untreue gemäß $§ 266$ StGB (Berlín: Duncker \& Humblot).

TURK, Austin (1969): Criminality and Legal Order (Chicago: Rand McNally).

USLANER, Eric (2008): Corruption, Inequality and the Rule of Law (Cambridge/Nueva York, Cambridge University Press).

WARREN, Mark E. (2004): "What Does Corruption Mean in a Democracy?", en: American Journal of Political Science (vol. 48 no. 2), pp. 328-43.

WILLIAMS, Glanville (1983): "Convictions and Fair Labelling", en: The Cambridge Law Journal (vol. 42 no. 1), pp. 85-95. 
Polít. Crim. Vol. 15, No 29 (Julio 2020), Art. 11, pp. 290-310

[http://politcrim.com/wp-content/uploads/2020/07/Vol15N29A11.pdf]

WOLFSLAST, Gabriele (1995): Staatlicher Strafanspruch und Verwirkung (Colonia/Berlín/Bonn/Múnich: Carl Heymanns).

ZYGLIDOPOULOS, Stelios; FLEMING, Peter; ROTHENBERG, Sandra (2009): "Rationalization, Overcompensation and the Escalation of Corruption in Organizations", en: Journal of Business Ethics (vol. 84 suplemento 1), pp. 65-73. 